\title{
SPECTRUM OF A LINEAR DIFFERENTIAL EQUATION OVER A FIELD OF FORMAL POWER SERIES
}

\author{
TINHINANE A. AZZOUZ
}

\begin{abstract}
In this paper we associate to a linear differential equation with coefficients in the field of Laurent formal power series a new geometric object, a spectrum in the sense of Berkovich. We compute this spectrum and show that it contains interesting informations about the equation.
\end{abstract}

\section{INTRODUCTION}

Over the centuries, the theory of differential equations represents an important field of mathematics. Notably, in the real and complex context, they encode behaviours of many physical phenomena. However, in ultrametric setting, they are rather related to algebraic and number theory problems. This part of the theory, appears around 1960, since Dwork's work on the variation of the Zeta function, and has become a central subject of investigation.

The study of ultrametric differential equations is more complicated than the usual context even for the linear case. Indeed, the solutions of an ultrametric linear differential equation may have finite radii of convergence, even without the presence of poles.

However, these radius behaves in a very controlled way, and their knowledge permits to obtain several informations about the equation. In particular, under some assumption, if a differential equation has two solutions with different radii, then it should correspond to a decomposition of the equation. This was firstly introduced by Dwork and mainly developed in the works of Robba [Rob75b],[Rob75c],[Rob75a], Dwork an Robba [DR77], Christol and Mebkhout[CM00], [CM01], Kedlaya[Ked10], [Ked13]. We point out that a large part of the literature is devoted to the two special cases: differential equation over a germ of a punctured disk, or Robba ring. In the special case of a trivial valued field $k$ of characteristic zero, the Robba ring corresponds exactly to the field of Laurent formal power series $k((T))$, and the radii of convergence of a differential equation over $k((T))$ are strongly related to the formal slopes (see for example [Pul15, Section 4.3]). The main fundamental decomposition and classification are expressed in the following way: Consider the differential field $\left(k((T)), T \frac{\mathrm{d}}{\mathrm{dT}}\right)$. We mean by a differential equation with coefficients in $k((T))$ a differential module $(M, \nabla)$ over $\left(k((T)), T \frac{\mathrm{d}}{\mathrm{dT}}\right)$.

(1) Decomposition theorem according to the slopes. Considering the $T$-adic valuation of the coefficients of the operator $\nabla$ in a cyclic basis, we can associate a Newton polygon, called formal. The formal slopes of $(M, \nabla)$ are the slopes of this polygon. The decomposition theorem is the following.

Theorem 0.1 ([DMR07, p. 97-107]). Let $\gamma_{1}<\cdots<\gamma_{\mu}$ be the slopes of the formal Newton polygon of $(M, \nabla)$, with multiplicity $n_{1}, \cdots, n_{\mu}$ respectively. Then

$$
(M, \nabla)=\bigoplus_{i=1}^{\mu}\left(M_{\gamma_{i}}, \nabla_{\gamma_{i}}\right)
$$

where $M_{\gamma_{i}}$ has dimension $n_{i}$ and a unique slope $\gamma_{i}$ with multiplicity $n_{i}$.

(2) The Turrittin-Levelt-Hukuhara decomposition theorem [Kat70], [Rob75a], [Ked10]. It claims that for any differential module $(M, \nabla)$, there exists a suitable finite extension $k^{\prime}\left(\left(T^{\frac{1}{n}}\right)\right)$ of $k((T))$ for 
which the pull-back of $(M, \nabla)$ with respect to this extension is an extension of differential modules of rank one.

This paper is a continuity of our work [Azz20], where we introduce, a new geometric invariante, the spectrum in the sense of Berkovich of a differential module, and develop some material for the computation of this one.

In this paper we focus on the computation of the spectrum of a differential module over $\left(k((T)), T \frac{\mathrm{d}}{\mathrm{dT}}\right)$, we show that it contains intersting information. More precisely, we can recover in one hand all the formal slopes of the differential module, in the other hand the exponentes of the regular part of the differential module. For this purpose we will use the classification results listed above. Before announcing the main result of the paper, we shall recall quickly the notion of the spectrum in the sense of Berkovich.

Recall that for an element $f$ of a non-zero $k$-algebra $E$ with unit, the classical spectrum of $f$ is the set

$$
\left\{a \in k ; f-a .1_{E} \text { is not invertible in } E\right\} .
$$

This set may be empty, even if $E$ is a $k$-Banach algebra. To deal with this issue Berkovich proposed to consider the spectrum not as a subset of $k$, but as a subset of the analytic affine line $\mathbb{A}_{k}^{1, \text { an }}$ (which is a bigger space than $k$ )[Ber90, Chapter 7]. Let $E$ be a non-zero $k$-Banach algebra and $f \in E$. The spectrum $\Sigma_{f, k}(E)$ of $f$ in the sense of Berkovich is the set of points of $\mathbb{A}_{k}^{1, \text { an }}$ that correspond to a pair $(\Omega, c)$, where $\Omega$ is a complete extension of $k$ and $c \in \Omega$, for which $f \otimes 1-1 \otimes c$ is not invertible in $E \hat{\otimes}_{k} \Omega$. This spectrum is non-empty, compact and satisfies other nice properties (cf.[Ber90, Theorem 7.1.2]).

Let $(M, \nabla)$ be a differential module over $\left(k((T)), T \frac{\mathrm{d}}{\mathrm{dT}}\right)$. Let $r \in(0,1)$ be a real positive number. From now on we endow $k((T))$ with the $T$-adic absolute value given by

$$
\left|\sum_{i \geq N} a_{i} T^{i}\right|:=r^{N}
$$

if $a_{N} \neq 0$. In this setting $k((T))$ is a complete valued field and the induced valuation on $k$ is the trivial valuation. From now on we endow $k$ with the trivial valuation. We can endow $M$ with $k((T))$-Banach structure, moreover it induces a $k$-Banach structure for which $\nabla: M \rightarrow M$ is a bounded operator. As in our previous work [Azz20], the spectrum $\Sigma_{\nabla, k}\left(\mathcal{L}_{k}(M)\right)$ of $(M, \nabla)$ will be the spectrum of $\nabla$ as an element of the $k$-Banach algebra $\mathcal{L}_{k}(M)$ of bounded endomorphism of $M$ with respect to operator norm.

Notice that we cannot use the classical index theorem of B. Malgrange [Mal74] to compute neither the spectrum in the sense of Berkovich nor the classical spectrum of $\nabla$. Indeed, it is relatively easy to show that any non trivial rank one connection on $k((T))$ is set-theoretically bijective. However, the set-theoretical inverse of the connection may not be bounded. This is due to the fact that the base field $k$ is trivially valued and Banach open mapping theorem does not hold in this setting.

For any positive real number $l$, we set $x_{0, l}$ to be the point of $\mathbb{A}_{k}^{1 \text {,an }}$ associated to $l$-Gauss norm on $k[T]$ (i.e. $\sum_{i} a_{i} T^{i} \mapsto \max _{i}\left|a_{i}\right| l^{i}$ ). The main result of the paper is the following:

Theorem 0.2. Assume that $k$ is algebraically closed. Let $r \in(0,1)$. Assume that $(k((S)),|\cdot|) \simeq$ $\left(\mathscr{H}\left(x_{0, r}\right),||.\right)$. Let $(M, \nabla)$ be a differential module over $\left(k((T)), T \frac{\mathrm{d}}{\mathrm{dT}}\right)$. Let $\left\{\gamma_{1}, \cdots, \gamma_{\nu}\right\}$ be the set of the slopes of $(M, \nabla)$ and let $\left\{a_{1}, \cdots, a_{\mu}\right\}$ be the set of the exponents of the regular part of $(M, \nabla)$. Then the spectrum of $\nabla$ as an element of $\mathcal{L}_{k}(M)$ is:

$$
\Sigma_{\nabla, k}\left(\mathcal{L}_{k}(M)\right)=\left\{x_{0, r^{-\gamma_{1}}}, \cdots, x_{0, r^{-\gamma_{\nu}}}\right\} \cup \bigcup_{i=1}^{\mu}\left(a_{i}+\mathbb{Z}\right) .
$$

This result clearly shows the importance of the points of the spectrum that are not in $k$. Indeed, form these points we can recover the slopes without multiplicity of the differential module. Therefore, we can say that the spectrum is highly connected not only to the smallest radius of convergence, which was the first motivation of our work, but to all radii of convergence of the solutions of a linear differential equation. 
On other hand, although differential modules over $\left(k((S)), S \frac{\mathrm{d}}{\mathrm{dS}}\right)$ are algebraic objects, their spectra in the sense of Berkovich depend highly on the choice of the absolute value on $k((S))$.

The paper is organized as follows. Section 1 is devoted to providing setting and notations. More precisely, we will recall the definition of the analytic affine line $\mathbb{A}_{k}^{1 \text {,an }}$, and give, in the setting of the paper, a precise topological description for disks and annuli of $\mathbb{A}_{k}^{1 \text {,an }}$. We will recall also the definition of the spectrum in the sense of Berkovich.

In Section 2, we introduce the spectrum of a differential module and recall some properties given in [Azz20]. We also explain the behavior of this spectrum after ramified ground field extension. In the end of the section we recall the definition of the formal Newton polygon.

Section 3 is devoted to announcing and proving the main result of the paper. Using the decomposition theorem according to the slopes, we can reduce the probleme to the computation of the spectrum of regular singular differential modules and differential modules without regular part. Since [Azz20, Proposition 3.15], in order to compute the spectrum of a regular singular module, it is enough to compute the spectrum of $T \frac{\mathrm{d}}{\mathrm{dT}}$ as an element of $\mathcal{L}_{k}(k((T)))$. For differential modules without regular part, we reduce the computation of the spectrum to the case of differential modules of rank one. This is possible by using Turrittin-Levelt-Hukura decomposition theorem and the behaviour of the spectrum after ramified ground field extension.

In the last section, we give a short discussion about the result and explain more precisely how it connect between two notions, the spectrum and radii of convergence.

Acknowledgments. The author wishes to express her gratitude to her advisors Andrea Pulita and Jérôme Poineau for their precious advice and suggestions, and for careful reading. She also thank Francesco Baldassarri, Frits Beukers, Antoine Ducros and Françoise Truc for useful occasional discussions and suggestions.

\section{Definitions And notations}

We will denote by $\mathbb{R}$ the field of real numbers, by $\mathbb{Z}$ the ring of integers and by $\mathbb{N}$ the set of natural numbers. We set $\mathbb{R}_{+}:=\left\{r \in \mathbb{R} ; r \in \mathbb{R}_{+}\right\}$.

In all the paper we fix $(k,||$.$) to be an algebraically closed field of characteristic 0$ equipped with a trivial valuation. Let $E(k)$ be the category whose objects are $\left(\Omega,|\cdot|_{\Omega}\right)$, where $\Omega$ is a field extension of $k$, complete with respect to the valuation $|\cdot|_{\Omega}$, and whose isomorphisms are isometric rings morphisms.

Analytic affin line and Berkovich spectrum. In order to get a better visualisation of the spectrum, we shall give an illustration of the analytic affine line.

Let $\Omega \in E(k)$. We consider $\Omega$-analytic spaces in the sense of Berkovich (see [Ber90]). We denote by $\mathbb{A}_{\Omega}^{1, \text { an }}$ the affine analytic line over the ground field $\Omega$. Recall that a point $x \in \mathbb{A}_{\Omega}^{1, \text { an }}$ corresponds to a multiplicative semi-norm $|\cdot|_{x}$ on $\Omega[T]$ (i.e. $|0|_{x}=0,|1|_{x}=1,|P-Q|_{x} \leq \max \left(|P|_{x},|Q|_{x}\right.$ ) and $|P \cdot Q|_{x}=|P|_{x} \cdot|Q|_{x}$ for all $\left.P, Q \in k[T]\right)$ whose restriction coincides with the absolute value of $\Omega$.

Notation 1.1. Let $x \in \mathbb{A}_{\Omega}^{1, \text { an }}$. We denote by $\mathscr{H}(x)$ the complete residue field associated to $x$.

Notation 1.2. Let $c \in \Omega$ and $r \in \mathbb{R}_{+}$. Denote by $x_{c, r}$ the point that corresponds to the semi-norm (norm if $r>0$ )

$$
\begin{aligned}
k[T] & \longrightarrow \mathbb{R}_{+} \\
\sum_{i=0}^{n} a_{i}(T-c)^{i} & \mapsto \max _{i}\left|a_{i}\right| r^{i} .
\end{aligned}
$$

Remark 1.3. Since $(k,||$.$) is trivially valued, any point of \mathbb{A}_{k}^{1, \text { an }}$ is a point of the form $x_{c, r}$. The points of the form $x_{c, 0}$ coincides with the element of $k$. 
For a point $x_{c, r} \in \mathbb{A}_{k}^{1 \text {,an }}$ with $r>0$ the field $\mathscr{H}\left(x_{c, r}\right)$ can be described more concretely. The case where $r=0$ is trivial, indeed $\mathscr{H}\left(x_{c, 0}\right) \simeq k$.

In the case where $r<1$, the field $\mathscr{H}\left(x_{c, r}\right)$ coincides with the field of Laurent formal power series

$$
k((T-c)):=\left\{\sum_{i \geq N} a_{i}(T-c)^{i} ; a_{i} \in k, N \in \mathbb{Z}\right\} .
$$

equipped with $(T-c)$-adic absolute value given by $\left|\sum_{i \geq N} a_{i}(T-c)^{i}\right|:=r^{N}$, if $a_{N} \neq 0$.

If $r>1$, then $\mathscr{H}\left(x_{c, r}\right)$ coincides with $k\left(\left((T-c)^{-1}\right)\right)$ equipped with the $(T-c)^{-1}$-adic absolute value given by $\left|\sum_{i \geq N} a_{i}(T-c)^{-i}\right|:=r^{-N}$, if $a_{N} \neq 0$.

Otherwise, $\mathscr{H}\left(x_{c, 1}\right)$ coincides with $k(T)$ equipped with the trivial absolute value. Let $\Omega \in E(k)$ and $c \in \Omega$. For $r \in \mathbb{R}_{+} \backslash\{0\}$ we set

and

$$
D_{\Omega}^{+}(c, r):=\left\{x \in \mathbb{A}_{\Omega}^{1, \text { an }} ;|T(x)-c| \leq r\right\}
$$

$$
D_{\Omega}^{-}(c, r):=\left\{x \in \mathbb{A}_{\Omega}^{1, \text { an }} ;|T(x)-c|<r\right\} .
$$

The point $x_{c, r} \in \mathbb{A}_{\Omega}^{1, \text { an }}$ corresponds to the disk $D_{\Omega}^{+}(c, r)$, more precisely it does not depend on the of the center $c$ (cf. [Ber90, Section 1.4.4]).

Let $c \in k$. The map

$$
[0,+\infty) \longrightarrow \mathbb{A}_{k}^{1, \text { an }}
$$

induces an homeomorphism between $[0,+\infty)$ and its image. Since the valuation is trivial on $k$, we can describe disks of $\mathbb{A}_{k}^{1, \text { an }}$ as follows.

Notation 1.4. We denote by $\left[x_{c, r}, \infty\right)\left(\operatorname{resp} .\left(x_{c, r}, \infty\right)\right)$ the image of $[r, \infty)(\operatorname{resp} .(r, \infty))$, by $\left[x_{c, r}, x_{c, r^{\prime}}\right]$ (resp. $\left.\left(x_{c, r}, x_{c, r^{\prime}}\right],\left[x_{c, r}, x_{c, r^{\prime}}\right),\left(x_{c, r}, x_{c, r^{\prime}}\right)\right)$ the image of $\left[r, r^{\prime}\right]$ (resp. $\left.\left(r, r^{\prime}\right],\left[r, r^{\prime}\right),\left(r, r^{\prime}\right)\right)$.

Let $c \in k$ and $r \in \mathbb{R}_{+}$. In the case where $r<1$, we have

$$
D_{k}^{+}(c, r)=\left[c, x_{c, r}\right] \quad D_{k}^{-}(c, r)=\left[c, x_{c, r}\right) .
$$

If $r>1$, recall that for all $a \in k, x_{a, 1}=x_{0,1}$ and we have

$$
\begin{gathered}
D_{k}^{+}(c, r)=\coprod_{a \in k}\left[a, x_{0,1}\right) \coprod\left[x_{0,1}, x_{0, r}\right] . \\
D_{k}^{-}(c, r)=\left\{\begin{array}{ll}
{\left[c, x_{0,1}\right)} & \text { if } r=1 \\
\coprod_{a \in k}\left[a, x_{0,1}\right) \amalg\left[x_{0,1}, x_{0, r}\right) & \text { otherwise }
\end{array} .\right.
\end{gathered}
$$

For $r_{1}, r_{2} \in \mathbb{R}_{+}$, such that $0<r_{1} \leq r_{2}$ we set

and for $r_{1}<r_{2}$ we set:

$$
C_{\Omega}^{+}\left(c, r_{1}, r_{2}\right):=D_{\Omega}^{+}\left(c, r_{2}\right) \backslash D_{\Omega}^{-}\left(c, r_{1}\right)
$$

$$
C_{\Omega}^{-}\left(c, r_{1}, r_{2}\right):=D_{\Omega}^{-}\left(c, r_{2}\right) \backslash D_{\Omega}^{+}\left(c, r_{1}\right) .
$$


We may suppress the index $\Omega$ when it is obvious from the context. $X$.

Let $X$ be an affinoid domain of $\mathbb{A}_{\Omega}^{1 \text {,an }}$, we denote by $\mathcal{O}(X)$ the $\Omega$-Banach algebra of global sections of

Since $k$ is trivially valued, if $r<1$ we have

$$
\mathcal{O}\left(D^{+}(c, r)\right)=k \llbracket T-c \rrbracket:=\left\{\sum_{i \in \mathbb{N}} a_{i}(T-c)^{i} ; a_{i} \in k\right\}
$$

otherwise,

$$
\mathcal{O}\left(D^{+}(c, r)\right)=k[T-c] .
$$

In the both cases, the multiplicative norm on $\mathcal{O}\left(D^{+}(c, r)\right)$ is :

$$
\left\|\sum_{i \in \mathbb{N}} a_{i}(T-c)^{i}\right\|=\max _{i \in \mathbb{N}}\left|a_{i}\right| r^{i}
$$

Let $X=C_{\Omega}^{+}\left(c, r_{1}, r_{2}\right)$

$$
\mathcal{O}\left(C_{\Omega}^{+}\left(c, r_{1}, r_{2}\right)\right)=\left\{\sum_{i \in \mathbb{N} \backslash\{0\}} \frac{a_{i}}{(T-c)^{i}} ; a_{i} \in \Omega,\left|a_{i}\right| r_{1}^{-i} \rightarrow 0\right\} \oplus \mathcal{O}\left(D^{+}\left(c, r_{2}\right)\right) .
$$

where $\left\|\sum_{i \in \mathbb{N} \backslash\{0\}} \frac{a_{i}}{(T-c)^{i}}\right\|=\max _{i}\left|a_{i}\right| r_{1}^{-i}$ and the sum above is equipped with the maximum norm.

Notation 1.5. Let $X$ be an analytic domain of $\mathbb{A}_{k}^{1 \text {,an }}$, and $f \in \mathcal{O}(X)$. We can see $f$ as an analytic map $X \rightarrow \mathbb{A}_{k}^{1, \text { an }}$ that we still denote by $f$.

Recall now the definition of the spectrum introduced by Berkovich.

Definition 1.6. Let $E$ be $k$-Banach algebra with unit and $f \in E$. The spectrum of $f$ is the set $\Sigma_{f, k}(E)$ of points $x \in \mathbb{A}_{k}^{1, \text { an }}$ such that the element $f \otimes 1-1 \otimes T(x)$ is not invertible in the $k$-Banach algebra $E \hat{\otimes}_{k} \mathscr{H}(x)$.

Remark 1.7. If there is no confusion we denote the spectrum of $f$, as an element of $E$, just by $\Sigma_{f}$.

Remark 1.8. The set $\Sigma_{f} \cap k$ coincides with the classical spectrum, i.e.

$$
\Sigma_{f} \cap k=\{a \in k ; f-a \text { is not invertible in } E\} .
$$

\section{Differential modules over $k((S))$ And SPeCtra}

In this section we recall some properties of differential module $(M, \nabla)$ over $(F, d)$, where $(F, d)$ is a finite differential extension of $\left(k((S)), S \frac{\mathrm{d}}{\mathrm{dS}}\right)$. We recall also the definition of the spectrum of a differential module $(M, \nabla)$ over $\left(k((S)), S \frac{\mathrm{d}}{\mathrm{dS}}\right)$, introduced in our previous work [Azz20], and explain more precisely its behavior under ramification of the indeterminate $S$.

Notation 2.1. Let $(F, d)$ be a differential field. We denote by $d$-Mod $(F)$ the category of differentiel modules over $(F, d)$ whose arrows are morphisms of differential modules.

Notation 2.2. Let $(F, d)$ be a differential field. We set $\mathscr{D}_{F}:=\bigoplus_{i \in \mathbb{N}} F \cdot D^{i}$ to be the ring of differential polynomials on $D$ with coefficients in $F$, where the multiplication is non-commutative and defined as follows: $D . f=d(f)+f . D$ for all $f \in A$. Let $P(D)=g_{0}+\cdots+g_{n-1} D^{n-1}+D^{n}$ be a monic differential polynomial. The quotient $\mathscr{D}_{F} / \mathscr{D}_{F} \cdot P(D)$ is an $F$-vector space of dimension $n$. Equipped with the multiplication by $D$, it is a differential module over $(F, d)$. 
Remark 2.3. Any differential module over a differential field $(F, d)$, with $d \neq 0$, is isomorphic to $\mathscr{D}_{F} / \mathscr{D}_{F} \cdot P(D)$, for some monic differential polynomial $P(D)$, thanks to the cyclic vector theorem.

Convention 2.4. We fix $r \in(0,1)$ and endow $k((S))$ with the $S$-adic absolute value given by $\left|\sum_{i \geq N} a_{i} S^{i}\right|:=$ $r^{N}$, if $a_{N} \neq 0$. In this setting the pair $(k((S)),||$.$) coincides with \mathscr{H}\left(x_{0, r}\right)$, where $x_{0, r} \in \mathbb{A}_{k}^{1, \text { an }}$.

2.1. Spectrum of a differential module. Recall that if $F$ is a finite extension of $k((S))$ of degree $m$, then we have $F \simeq k\left(\left(S^{\frac{1}{m}}\right)\right)$ [VS12, Proposition 3.3]. The absolute value |.| on $k((S))$ extends uniquely to an absolute value on $F$. The pair $(F,||$.$) is an element of E(k)$ and can be identified with $\mathscr{H}\left(x_{0, r \frac{1}{m}}\right)$. Moreover, the derivation $S \frac{\mathrm{d}}{\mathrm{dS}}$ extends uniquely to a bounded derivation $d$ on $F$, satisfying $d\left(S^{\frac{1}{m}}\right)=\frac{1}{m} S^{\frac{1}{m}}$. Conversely, any finite differential extension field of $\left(k((S)), S \frac{\mathrm{d}}{\mathrm{dS}}\right)$ is obtained in this way.

We shall recall quickly the construction of the spectrum of a differential module, which is introduced more precisely in [Azz20, Section 3.2]. Let $(M, \nabla)$ be a differential module over some differential extension field $(F, d)$ of $\left(k((S)), S \frac{\mathrm{d}}{\mathrm{dS}}\right)$. The $F$-vector space $M$ can be endowed with a structure of $F$-Banach space, unique up to bi-bouned isomorphism of $F$-Banach space. In this sitting, the operator $\nabla$ can be seen as an element of $\left(\mathcal{L}_{k}(M),\|\cdot\|_{\mathrm{op}}\right)$, the $k$-Banach algebra of bounded $k$-endomorphisms of $M$ with respect to the operator norm. The spectrum of $(M, \nabla)$ is the spectrum of $\nabla$ as an element of $\mathcal{L}_{k}(M)$, that we denote by $\Sigma_{\nabla, k}\left(\mathcal{L}_{k}(M)\right)$ (or just $\Sigma_{\nabla}$ if it is obvious from the context). This spectrum is a compact nonempty set, moreover the smallest closed disk centring in zero containing $\Sigma_{\nabla, k}\left(\mathcal{L}_{k}(M)\right)$ has radius equal to $\|\nabla\|_{\mathrm{Sp}}=\lim _{n \rightarrow+\infty}\|\nabla\|_{\mathrm{oP}}^{\frac{1}{m}}($ see $[$ Ber90, Theorem 7.1.2]). Stressing also that this spectrum is invariant by bi-bounded isomorphisms of differential modules.

However, it depends on the choice of the derivation. We will see further that $S \frac{\mathrm{d}}{\mathrm{dS}}$ has a good behavior under ramification of the indeterminate, which makes the choice of $S \frac{\mathrm{d}}{\mathrm{dS}}$ more conviniente for the computation of the spectrum.

We need now to recall importante materials developed in our previous work [Azz20], that are very necessary to the computation of the spectrum.

Proposition 2.5. Let $(M, \nabla),\left(M_{1}, \nabla_{1}\right)$ and $\left(M_{2}, \nabla_{2}\right)$ be three differential modules over $(F, d)$. If we have two exact sequences of the form:

$$
\begin{aligned}
& 0 \rightarrow\left(M_{1}, \nabla_{1}\right) \rightarrow(M, \nabla) \rightarrow\left(M_{2}, \nabla_{2}\right) \rightarrow 0 \\
& 0 \rightarrow\left(M_{2}, \nabla_{2}\right) \rightarrow(M, \nabla) \rightarrow\left(M_{1}, \nabla_{1}\right) \rightarrow 0 .
\end{aligned}
$$

Then we have $\Sigma_{\nabla}\left(\mathcal{L}_{k}(M)\right)=\Sigma_{\nabla_{1}}\left(\mathcal{L}_{k}\left(M_{1}\right)\right) \cup \Sigma_{\nabla_{2}}\left(\mathcal{L}_{k}\left(M_{2}\right)\right)$.

Proof. See [Azz20, Proposition 3.7] and [Azz20, Remark 3.8].

Corollary 2.6. Let $(M, \nabla),\left(M_{1}, \nabla_{1}\right)$ and $\left(M_{2}, \nabla_{2}\right)$ be three differential modules over $(F, d)$. If we suppose that $(M, \nabla)=\left(M_{1}, \nabla_{1}\right) \oplus\left(M_{2}, \nabla_{2}\right)$, then we have $\Sigma_{\nabla}\left(\mathcal{L}_{k}(M)\right)=\Sigma_{\nabla_{1}}\left(\mathcal{L}_{k}\left(M_{1}\right)\right) \cup \Sigma_{\nabla_{2}}\left(\mathcal{L}_{k}\left(M_{2}\right)\right)$.

Corollary 2.7. Let $f \in F$. Consider the differential module $(M, \nabla):=\left(\mathscr{D}_{F} / \mathscr{D}_{F} \cdot(D-f)^{n}, D\right)$. Then we have:

$$
\Sigma_{\nabla}\left(\mathcal{L}_{k}(M)\right)=\Sigma_{S \frac{\mathrm{d}}{\mathrm{dS}}+f}\left(\mathcal{L}_{k}(F)\right) .
$$

2.2. Spectrum of a differential module after ramified ground field extension. In this section we show how the spectrum of a differential module behaves after ramified ground field extensions.

Let $(F, d)$ be a finite differential extension of $\left(k((S)), S \frac{\mathrm{d}}{\mathrm{dS}}\right)$. Let $m \in \mathbb{N}$ such that $F \simeq k\left(\left(S^{\frac{1}{m}}\right)\right)$. Now, if we set $Z=S^{\frac{1}{m}}$, then we have $F=k((Z))$ and $d=\frac{Z}{m} \frac{\mathrm{d}}{\mathrm{dZ}}$. Note that we can see $\left(F, \frac{Z}{m} \frac{\mathrm{d}}{\mathrm{dZ}}\right)$ as a differential module over $\left(k((S)), S \frac{\mathrm{d}}{\mathrm{dS}}\right)$. In the basis $\left\{1, Z, \cdots, Z^{m-1}\right\}$ we have: 


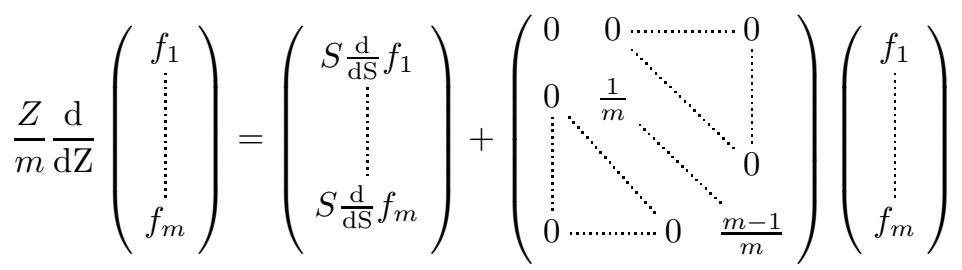

We have a functor:

$$
\begin{aligned}
I_{F}{ }^{*}: S \frac{\mathrm{d}}{\mathrm{dS}}-\operatorname{Mod}(k((S))) & \longrightarrow \frac{Z}{m} \frac{\mathrm{d}}{\mathrm{dZ}}-\operatorname{Mod}(F) \\
(M, \nabla) & \mapsto\left(I_{F}^{*} M, I_{F}{ }^{*} \nabla\right)
\end{aligned}
$$

where $I_{F}{ }^{*} M=M \otimes_{k((S))} F$ and the connection $I_{F}{ }^{*} \nabla$ is defined as follows:

$$
I_{F}^{*} \nabla=\nabla \otimes 1+1 \otimes \frac{Z}{m} \frac{\mathrm{d}}{\mathrm{dZ}}
$$

Let $(M, \nabla)$ be an object of $S \frac{\mathrm{d}}{\mathrm{dS}}-\operatorname{Mod}(k((S)))$ of rank $n$. If $\left\{e_{1}, \ldots, e_{n}\right\}$ is a basis of $M$ such that we have:

$$
\nabla\left(\begin{array}{c}
f_{1} \\
\vdots \\
f_{n}
\end{array}\right)=\left(\begin{array}{c}
S \frac{\mathrm{d}}{\mathrm{dS}} f_{1} \\
\vdots \\
S \frac{\mathrm{d}}{\mathrm{dS}} f_{n}
\end{array}\right)+G\left(\begin{array}{c}
f_{1} \\
\vdots \\
f_{n}
\end{array}\right),
$$

with $G \in \mathcal{M}(k((S)))$, then $\left(I_{F}{ }^{*} M, I_{F}{ }^{*} \nabla\right)$ is of rank $n$ and in the basis $\left\{e_{1} \otimes 1, \ldots, e_{n} \otimes 1\right\}$ we have:

$$
I_{F}{ }^{*} \nabla\left(\begin{array}{c}
f_{1} \\
\vdots \\
f_{n}
\end{array}\right)=\left(\begin{array}{c}
\frac{Z}{m} \frac{\mathrm{d}}{\mathrm{dZ}} f_{1} \\
\vdots \\
\frac{Z}{m} \frac{\mathrm{d}}{\mathrm{dZ}} f_{n}
\end{array}\right)+G\left(\begin{array}{c}
f_{1} \\
\vdots \\
f_{n}
\end{array}\right) .
$$

We have also the functor:

$$
\begin{aligned}
I_{F *}: \frac{Z}{m} \frac{\mathrm{d}}{\mathrm{dZ}}-\operatorname{Mod}(F) & \longrightarrow S \frac{\mathrm{d}}{\mathrm{dS}}-\operatorname{Mod}(k((S))) \\
(M, \nabla) & \mapsto \quad\left(I_{F *} M, I_{F *} \nabla\right)
\end{aligned}
$$

where $I_{F *} M$ is the restriction of scalars of $M$ via $k((S)) \hookrightarrow F$, and $\nabla=I_{F *} \nabla$ are equal as $k$-linear maps. If $(M, \nabla)$ has rank equal to $n$, the rank of $\left(I_{F *} M, I_{F *} \nabla\right)$ is equal to $n . m$.

Let $(M, \nabla)$ be an object of $S \frac{\mathrm{d}}{\mathrm{dS}}-\operatorname{Mod}(k((S)))$ of rank $n$. The differential module $\left(I_{F *} I_{F}{ }^{*} M, I_{F *} I_{F}{ }^{*} \nabla\right)$ has rank $n m$. Let $\left\{e_{1}, \cdots, e_{n}\right\}$ be a basis of $(M, \nabla)$ and let $G$ be the associated matrix in this basis. Then the matrix associated to $\left(I_{F *} I_{F}{ }^{*} M, I_{F *} I_{F}{ }^{*} \nabla\right)$ in the basis $\left\{e_{1} \otimes 1, \cdots, e_{n} \otimes 1, e_{1} \otimes Z, \cdots, e_{n} \otimes\right.$ $\left.Z, \cdots, e_{1} \otimes Z^{m-1}, \cdots, e_{n} \otimes Z^{m-1}\right\}$ is:

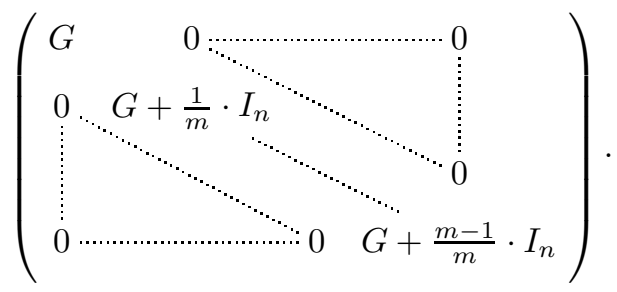

Therefore we have the following isomorphism:

$$
\left(I_{F *} I_{F}{ }^{*} M, I_{F *} I_{F}{ }^{*} \nabla\right) \simeq \bigoplus_{i=0}^{m-1}\left(M, \nabla+\frac{i}{m}\right)
$$


As $k$-Banach spaces $I_{F *} I_{F}{ }^{*} M$ and $I_{F}{ }^{*} M$ are the same, and $I_{F *} I_{F}{ }^{*} \nabla$ as a $k$-linear map coincides with $I_{F}{ }^{*} \nabla$. Therefore, we have

$$
\Sigma_{I_{F}^{*} \nabla, k}\left(\mathcal{L}_{k}\left(I_{F}^{*} M\right)\right)=\Sigma_{I_{F *} I_{F}^{*} \nabla, k}\left(\mathcal{L}_{k}\left(I_{F *} I_{F}^{*} M\right)\right) .
$$

By [Ber90, Proposition7.1.4] and Corollary 2.6 we have:

$$
\Sigma_{I_{F}{ }^{*} \nabla, k}\left(\mathcal{L}_{k}\left(I_{F}{ }^{*} M\right)\right)=\bigcup_{i=0}^{m-1} \frac{i}{m}+\Sigma_{\nabla, k}\left(\mathcal{L}_{k}(M)\right)^{1} .
$$

2.3. Newton polygon and the decomposition according to the slopes. Let $v: k\left(\left(S^{\frac{1}{m}}\right)\right) \rightarrow \mathbb{Z} \cup\{\infty\}$ be the valuation map associated to the $S^{\frac{1}{m}}$-adic valuation.

Let $P=\sum_{i=0}^{n} g_{i} D^{i}$ be an element of $\mathscr{D}_{k((S))}$. Let $L_{P}$ be the convex hull in $\mathbb{R}^{2}$ of the set of points

$$
\left\{\left(i, v\left(g_{i}\right)\right) \mid 0 \leq i \leq n\right\} \cup\left\{\left(0, \min _{0 \leq i \leq n} v\left(g_{i}\right)\right)\right\} .
$$

Definition 2.8 ([VS12, Definition 3.44]). The Newton polygon $\mathrm{NP}(P)$ of $P$ is the boundary of $L_{P}$. The finite slopes $\gamma_{i}$ of $P$ are called the slopes of $\mathrm{NP}(P)$. The horizontal width of the segment of $N P(P)$ of slope $\gamma_{i}$ is called the multiplicity of $\gamma_{i}$.

Definition 2.9. A differential module $(M, \nabla)$ over $\left(k((S)), S \frac{\mathrm{d}}{\mathrm{dS}}\right)$ is said to be regular singular if there exists a basis for which the associated matrix $G$ has constant entries (i.e $G \in \mathcal{M}_{n}(k)$ ). We will call the eigenvalues of such $G$ the exponents of $(M, \nabla)$.

Proposition 2.10. Let $(M, \nabla)$ be a differential module over $\left(k((S)), S \frac{\mathrm{d}}{\mathrm{dS}}\right)$. The following properties are equivalent:

- $(M, \nabla)$ is regular singular;

- There exists a differential polynomial $P(D)$ with only one slope equal to 0 such that $(M, \nabla) \simeq$ $\mathscr{D}_{k((S))} / \mathscr{D}_{k((S))} \cdot P(D)$;

- There exists $P(D)=g_{0}+g_{1} D+\cdots+g_{n-1} D^{n-1}+D^{n}$ with $g_{i} \in k \llbracket S \rrbracket$, such that $(M, \nabla) \simeq$ $\left(\mathscr{D}_{k((S))} / \mathscr{D}_{k((S))} \cdot P(D), D\right)$;

- There exists $P(D)=g_{0}+g_{1} D+\cdots+g_{n-1} D^{n-1}+D^{n}$ with $g_{i} \in k$, such that $(M, \nabla) \simeq$ $\left(\mathscr{D}_{k((S))} / \mathscr{D}_{k((S))} \cdot P(D), D\right)$;

Proof. See [Ked10, Corollary 7.1.3] and [Chr, Proposition 10.1].

Proposition 2.11 ([Ked10, Proposition 7.3.6]). Let $P(D)=g_{0}+g_{1} D+\cdots+g_{n-1} D^{n-1}+D^{n}$ such that $g_{i} \in k \llbracket S \rrbracket$. Then we have the isomorphism in $S \frac{\mathrm{d}}{\mathrm{dS}}-\operatorname{Mod}(k((S)))$ :

$$
\left(\mathscr{D}_{k((S))} / \mathscr{D}_{k((S))} \cdot P(D), D\right) \simeq\left(\mathscr{D}_{k((S))} / \mathscr{D}_{k((S))} \cdot P_{0}(D)\right),
$$

where $P_{0}(D)=g_{0}(0)+g_{1}(0) D+\cdots g_{n-1}(0) D^{n-1}+D^{n}$.

Remark 2.12. This proposition means in particular that for all $f \in k \llbracket S \rrbracket$ there exists $g \in k((S)) \backslash\{0\}$ such that $f-\frac{S \frac{\mathrm{d}}{\mathrm{dS}}(g)}{g}=f(0)$. Indeed, by Proposition 2.11 we have $\left(k((S)), S \frac{\mathrm{d}}{\mathrm{dS}}+f\right) \simeq\left(k((S)), S \frac{\mathrm{d}}{\mathrm{dS}}+f(0)\right)$. This is equivalente to saying that there exists $g \in k((S)) \backslash\{0\}$ such that

$$
g^{-1} \circ S \frac{\mathrm{d}}{\mathrm{dS}} \circ g+f=S \frac{\mathrm{d}}{\mathrm{dS}}+f(0) \text {. }
$$

Definition 2.13. Let $(M, \nabla)$ be an element of $S \frac{\mathrm{d}}{\mathrm{dS}}-\operatorname{Mod}(k((S)))$, and let $P(D) \in \mathscr{D}_{k((S))}$ such that $(M, \nabla) \simeq\left(\mathscr{D}_{k((S))} / \mathscr{D}_{k((S))} \cdot P(D), D\right)$. If all the slopes of $P(D)$ are different from 0 , then we say that $(M, \nabla)$ is without regular part.

\footnotetext{
${ }^{1}$ This is the image of the spectrum by the polynomial function $\frac{i}{m}+T$.
} 
Proposition 2.14. Let $(M, \nabla)$ be a differential module over $\left(k((S)), S \frac{\mathrm{d}}{\mathrm{dS}}\right)$. Then we have decomposition in $S \frac{\mathrm{d}}{\mathrm{dS}}-\operatorname{Mod}(k((S)))$ :

$$
(M, \nabla)=\left(M_{\text {reg }}, \nabla_{\text {reg }}\right) \oplus\left(M_{\text {irr }}, \nabla_{\text {irr }}\right)
$$

where $\left(M_{\mathrm{reg}}, \nabla_{\mathrm{reg}}\right)$ is a regular singular differential module and $\left(M_{\mathrm{irr}}, \nabla_{\mathrm{irr}}\right)$ is a differential module without regular part.

Proof. See [Chr, Proposition 12.1] and [VS12, Theorem 3.48].

\section{Spectrum of a differential module}

In this section we compute the spectrum of a differential module $(M, \nabla)$ over $\left(k((S)), S \frac{\mathrm{d}}{\mathrm{dS}}\right)$. The main statement of the paper is the following.

Theorem 3.1. Let $r \in(0,1)$. Assume that $(k((S)),||.) \simeq\left(\mathscr{H}\left(x_{0, r}\right),||.\right)$. Let $(M, \nabla)$ be a differential module over $\left(k((S)), S \frac{\mathrm{d}}{\mathrm{dS}}\right)$. Let $\left\{\gamma_{1}, \cdots, \gamma_{\nu}\right\}$ be the set of slopes of $(M, \nabla)$ and let $\left\{a_{1}, \cdots, a_{\mu}\right\}$ be the set of exponents of the regular part of $(M, \nabla)$. Then the spectrum of $\nabla$ as an element of $\mathcal{L}_{k}(M)$ is:

$$
\Sigma_{\nabla, k}\left(\mathcal{L}_{k}(M)\right)=\left\{x_{0, r^{-\gamma_{1}}}, \cdots, x_{0, r^{-\gamma_{\nu}}}\right\} \cup \bigcup_{i=1}^{\mu}\left(a_{i}+\mathbb{Z}\right)
$$

Remark 3.2. We observe that although differential modules over $\left(k((S)), S \frac{\mathrm{d}}{\mathrm{dS}}\right)$ are algebraic objects, their spectra in the sense of Berkovich depends highly on the choice of the absolute value on $k((S))$.

According to Corollary 2.14 we have the decomposition:

$$
(M, \nabla)=\left(M_{\text {reg }}, \nabla_{\text {reg }}\right) \oplus\left(M_{\text {irr }}, \nabla_{\text {irr }}\right)
$$

We know that $\Sigma_{\nabla}=\Sigma_{\nabla_{\text {reg }}} \cup \Sigma_{\nabla_{\text {irr }}}$ (cf. Proposition 2.6). Therefore, in order to obtain the main statement, it is enough to know the spectrum of a regular singular differential module and the spectrum of differential module without regular part.

3.1. Spectrum of regular singular differential module. Let $(M, \nabla)$ be a regular singular differential module. We point out, since our work in [Azz20], that the difficulty of the computation of the spectrum of $\nabla$ is reduced to the computation of $\Sigma_{S \frac{\mathrm{d}}{\mathrm{dS}}, k}\left(\mathcal{L}_{k}(k((S)))\right)$. This is due, more precisely, to the following Proposition.

Proposition 3.3 ([Azz20, Proposition 3.15]). Let $(M, \nabla)$ be a differential module over $\left(k((S))\right.$ ), $\left.S \frac{\mathrm{d}}{\mathrm{dS}}\right)$ such that:

$$
\nabla\left(\begin{array}{c}
f_{1} \\
\vdots \\
f_{n}
\end{array}\right)=\left(\begin{array}{c}
d f_{1} \\
\vdots \\
d f_{n}
\end{array}\right)+G\left(\begin{array}{c}
f_{1} \\
\vdots \\
f_{n}
\end{array}\right),
$$

with $G \in \mathcal{M}_{n}(k)$. The spectrum of $\nabla$ is $\Sigma_{\nabla, k}\left(\mathcal{L}_{k}(M)\right)=\bigcup_{i=1}^{N}\left(a_{i}+\Sigma_{S \frac{\mathrm{d}}{\mathrm{dS}}, k}\left(\mathcal{L}_{k}(k((S)))\right)\right)$, where $\left\{a_{1}, \ldots, a_{N}\right\}$ are the eigenvalues of $G$.

The behaviour of the spectrum of $\nabla$ is recapitulated in the following theorem.

Theorem 3.4. Let $(M, \nabla)$ be a regular singular differential module over $\left(k((S)), S \frac{\mathrm{d}}{\mathrm{dS}}\right)$. Let $G$ a matrix associated to $\nabla$ with constant entries (i.e. $G \in \mathcal{M}_{\nu}(k)$ ), and let $\left\{a_{1}, \cdots, a_{N}\right\}$ be the set of eigenvalues of $G$. The spectrum of $\nabla$ is

$$
\Sigma_{\nabla, k}\left(\mathcal{L}_{k}(M)\right)=\bigcup_{i=1}^{N}\left(a_{i}+\mathbb{Z}\right) \cup\left\{x_{0,1}\right\} .
$$


Lemma 3.5 ([VS12, Proposition 3.12]). There exists a basis for which the set of the eigenvalues $\left\{a_{1}, \cdots, a_{N}\right\}$ of $G$ satisfies $a_{i}-a_{j} \notin \mathbb{Z}$ for each $i \neq j$.

We now compute the spectrum of $S \frac{\mathrm{d}}{\mathrm{dS}}$.

Lemma 3.6. The norm and spectral semi-norm of $S \frac{\mathrm{d}}{\mathrm{dS}}$ as an element of $\mathcal{L}_{k}(k((S)))$ satisfy:

$$
\left\|S \frac{\mathrm{d}}{\mathrm{dS}}\right\|=1, \quad\left\|S \frac{\mathrm{d}}{\mathrm{dS}}\right\|_{\mathrm{Sp}}=1 .
$$

Proof. Since $\|S\|=|S|=r$ and $\left\|\frac{\mathrm{d}}{\mathrm{dS}}\right\|=\frac{1}{r}$ (cf. [Pul15, Lemma 4.4.1]), we have $\left\|S \frac{\mathrm{d}}{\mathrm{dS}}\right\| \leq 1$. Hence also, $\left\|S \frac{\mathrm{d}}{\mathrm{dS}}\right\|_{\mathrm{Sp}} \leq 1$. The map

$$
\begin{aligned}
\mathcal{L}_{k}(\mathscr{H}(x)) & \longrightarrow \mathcal{L}_{k}(\mathscr{H}(x)) \\
\varphi & \mapsto S^{-1} \circ \varphi \circ S
\end{aligned}
$$

is bi-bounded and induces change of basis. Therefore, as $S^{-1} \circ\left(S \frac{\mathrm{d}}{\mathrm{dS}}\right) \circ S=S \frac{\mathrm{d}}{\mathrm{dS}}+1$, we have $\left\|S \frac{\mathrm{d}}{\mathrm{dS}}\right\|_{\mathrm{Sp}}=$ $\left\|S \frac{\mathrm{d}}{\mathrm{dS}}+1\right\|_{\mathrm{Sp}}$. Since 1 commutes with $S \frac{\mathrm{d}}{\mathrm{dS}}$, we have:

$$
1=\|1\|_{\mathrm{Sp}}=\left\|S \frac{\mathrm{d}}{\mathrm{dS}}+1-S \frac{\mathrm{d}}{\mathrm{dS}}\right\|_{\mathrm{Sp}} \leq \max \left(\left\|S \frac{\mathrm{d}}{\mathrm{dS}}+1\right\|_{\mathrm{Sp}},\left\|S \frac{\mathrm{d}}{\mathrm{dS}}\right\|_{\mathrm{Sp}}\right) .
$$

Consequently, we obtain

$$
\left\|S \frac{\mathrm{d}}{\mathrm{dS}}\right\|=\left\|S \frac{\mathrm{d}}{\mathrm{dS}}\right\|_{\mathrm{Sp}}=1 .
$$

Proposition 3.7. The spectrum of $S \frac{\mathrm{d}}{\mathrm{dS}}$ as an element of $\mathcal{L}_{k}(k((S)))$ is equal to:

$$
\Sigma_{S \frac{\mathrm{d}}{\mathrm{dS}}}\left(\mathcal{L}_{k}(k((S)))\right)=\mathbb{Z} \cup\left\{x_{0,1}\right\}
$$

Proof. We set $d:=S \frac{\mathrm{d}}{\mathrm{dS}}$ and $\Sigma_{d-n}:=\Sigma_{d-n, k}\left(\mathcal{L}_{k}(k((S)))\right)$. As $\|d\|_{\mathrm{Sp}}=1$ (cf. Lemma 3.6), we have $\Sigma_{d} \subset D^{+}(0,1)$.

Let $a \in k \cap D^{+}(0,1)$. If $a \in \mathbb{Z}$, then we have $(d-a)\left(S^{a}\right)=0$. Hence, $d-a$ is not injective and $\mathbb{Z} \subset \Sigma_{d}$. As the spectrum is compact, we have $\mathbb{Z} \cup\left\{x_{0,1}\right\} \subset \Sigma_{d}$. If $a \notin \mathbb{Z}$, then $d-a$ is invertible in $\mathcal{L}_{k}(k((S)))$. Indeed, let $g(S)=\sum_{i \in \mathbb{Z}} b_{i} S^{i} \in k((S))$. If there exists $f=\sum_{i \in \mathbb{Z}} a_{i} S^{i} \in k((S))$ such that $(d-a) f=g$, then for each $i \in \mathbb{Z}$ we have

$$
a_{i}=\frac{b_{i}}{(i-a)}
$$

For each $i \in \mathbb{Z}$ we have $\left|a_{i}\right|=\left|b_{i}\right|$. This means that $f$ it is unique and converges in $k((S))$. We obtain also $|f|=|g|$. Consequently, the set theoretical inverse $(d-a)^{-1}$ is bounded and $\left\|(d-a)^{-1}\right\|=1$. Hence, we have $\left\|(d-a)^{-1}\right\|_{\mathrm{Sp}}=1$. According to [Azz20, Lemma 2.20], we have $D^{-}(a, 1) \subset \mathbb{A}_{k}^{1, \text { an }} \backslash \Sigma_{d}$.

Recall that $D^{+}(0,1)=\bigcup_{a \in k}\left[a, x_{0,1}\right]$ (cf. (1.4)). In order to end the proof, it is enough to show that $\left(n, x_{0,1}\right) \subset \mathbb{A}_{k}^{1, \text { an }} \backslash \Sigma_{d}$ for all $n \in \mathbb{Z}$. Let $n \in \mathbb{Z}$. Then we have

$$
k((S))=k . S^{n} \oplus \widehat{\bigoplus}_{i \in \mathbb{Z} \backslash\{n\}} k \cdot S^{i} .
$$

The operator $(d-n)$ stabilises both $k \cdot S^{n}$ and $\widehat{\bigoplus}_{i \in \mathbb{Z} \backslash\{n\}} k \cdot S^{i}$. We set $\left.(d-n)\right|_{k \cdot S^{n}}=\nabla_{1}$ and $(d-$ $n)\left.\right|_{\widehat{\oplus}_{i \in \mathbb{Z} \backslash\{n\}} k . S^{i}}=\nabla_{2}$. We set $\Sigma_{\nabla_{1}}:=\Sigma_{\nabla_{1}, k}\left(\mathcal{L}_{k}\left(k . S^{n}\right)\right)$ and $\Sigma_{\nabla_{2}}:=\Sigma_{\nabla_{2}, k}\left(\mathcal{L}_{k}\left(\widehat{\bigoplus}_{i \in \mathbb{Z} \backslash\{n\}} k . S^{i}\right)\right)$. We have $\nabla_{1}=0$. By [Azz20, Lemma 2.29], we have:

$$
\Sigma_{d-n}=\Sigma_{\nabla_{1}} \cup \Sigma_{\nabla_{2}}=\{0\} \cup \Sigma_{\nabla_{2}} .
$$

We now prove that

$$
D^{-}(0,1) \cap \Sigma_{\nabla_{2}}=\varnothing \text {. }
$$


The operator $\nabla_{2}$ is invertible in $\mathcal{L}_{k}\left(\widehat{\bigoplus}_{i \in \mathbb{Z} \backslash\{n\}} k . S^{i}\right)$. Indeed, let $g(S)=\sum_{i \in \mathbb{Z} \backslash\{n\}} b_{i} S^{i} \in \widehat{\bigoplus}_{i \in \mathbb{Z} \backslash\{n\}} k . S^{i}$. If there exists $f=\sum_{i \in \mathbb{Z} \backslash\{n\}} a_{i} S^{i} \in \widehat{\bigoplus}_{i \in \mathbb{Z} \backslash\{n\}} k . S^{i}$ such that $\nabla_{2}(f)=g$, then for each $i \in \mathbb{Z} \backslash\{n\}$ we have

$$
a_{i}=\frac{b_{i}}{(i-n)} .
$$

Since $\left|a_{i}\right|=\left|b_{i}\right|$, the element $f$ exists and it is unique, moreover $|f|=|g|$. Hence, $\nabla_{2}$ is invertible in $\mathcal{L}_{k}\left(\widehat{\oplus}_{i \in \mathbb{Z} \backslash\{n\}} k . S^{i}\right)$ and as a $k$-linear map it is isometric. Therefore, we have $\left\|\nabla_{2}^{-1}\right\|_{\text {Sp }}=1$. Hence, by [Azz20, Lemma 2.20] $D^{-}(0,1) \subset \mathbb{A}_{k}^{1, \text { an }} \backslash \Sigma_{\nabla_{2}}$. Consequently, $D^{-}(0,1) \cap \Sigma_{d-n}=\{0\}$. As $\Sigma_{d}=\Sigma_{d-n}+n$ (cf. [Ber90, Proposition7.1.4]), we have $D^{-}(n, 1) \cap \Sigma_{d}=\{n\}$. Therefore, for all $n \in \mathbb{Z}$ we have $\left(n, x_{0,1}\right) \subset$ $\mathbb{A}_{k}^{1, \text { an }} \backslash \Sigma_{d}$ and the claim follows.

3.2. Spectrum of a differential module without regular part. Recall the following Theorem, which is the celebrated theorem of Turrittin. It ensures that any differential module becomes extension of rank one differential modules after pull-back by a suitable ramified extension.

Theorem 3.8 ([Tur55]). Let $(M, \nabla)$ be a differential module over $\left(k((S)), S \frac{\mathrm{d}}{\mathrm{dS}}\right)$. There exists a finite extension $F=k\left(\left(S^{\frac{1}{m}}\right)\right)$ such that we have:

$$
\left(I_{F}{ }^{*} M, I_{F}{ }^{*} \nabla\right)=\bigoplus_{i=1}^{N}\left(\mathscr{D}_{F} / \mathscr{D}_{F} \cdot\left(D-f_{i}\right)^{\alpha_{i}}, D\right)
$$

where $f_{i} \in k \llbracket S^{-\frac{1}{m}} \rrbracket$ and $\alpha_{i} \in \mathbb{N}$.

Proof. See [VS12, Theorem 3.1].

Now, in order to compute the spectrum, we need the following result.

Proposition 3.9. Let $f=\sum_{i \in \mathbb{Z}} a_{i} S^{\frac{i}{m}}$ an element of $F:=k\left(\left(S^{\frac{1}{m}}\right)\right)$ and let $(F, \nabla)$ be the differential module of rank one such that $\nabla=S \frac{\mathrm{d}}{\mathrm{dS}}+f$. If $v(f)<0$, then the spectrum of $\nabla$ as an element of $\mathcal{L}_{k}(F)$ is:

$$
\Sigma_{\nabla, k}\left(\mathcal{L}_{k}(F)\right)=\left\{x_{0, r v(f)}\right\} .
$$

The following results are necessary to prove this proposition.

Lemma 3.10. Let $\Omega \in E(k)$. Consider the isometric embedding of $k$-algebras

$$
\begin{aligned}
\Omega & \longrightarrow \mathcal{L}_{k}(\Omega) \\
a & \mapsto b \mapsto a . b
\end{aligned}
$$

With respect to this embedding, $\Omega$ is a maximal commutative subalgebra of $\mathcal{L}_{k}(\Omega)$.

Proof. Let $A$ be a commutative subalgebra of $\mathcal{L}_{k}(\Omega)$ such that $\Omega \subset A$. Then each element of $A$ is an endomorphism of $\Omega$ that commutes with the elements of $\Omega$. Therefore, $A \subset \mathcal{L}_{\Omega}(\Omega)=\Omega$. Hence, we have $A=\Omega$.

Lemma 3.11. Let $\Omega \in E(k)$ and $\pi_{\Omega / k}: \mathbb{A}_{\Omega}^{1 \text {,an }} \rightarrow \mathbb{A}_{k}^{1 \text {,an }}$ be the canonical projection. Let $\alpha \in \Omega$. The spectrum of $\alpha$ as an element of $\mathcal{L}_{k}(\Omega)$ is $\Sigma_{\alpha}\left(\mathcal{L}_{k}(\Omega)\right)=\left\{\pi_{\Omega / k}(\alpha)\right\}$.

Proof. By [Ber90, Proposition 7.1.4, i)], the spectrum of $\alpha$ as an element of $\Omega$ is the point which corresponds to the character $k[T] \rightarrow \Omega, T \mapsto \alpha$. Hence, $\Sigma_{\alpha, k}(\Omega)=\left\{\pi_{\Omega / k}(\alpha)\right\}$. By Lemma 3.10 and [Ber90, Proposition 7.2.4] we conclude. 
Proof of Proposition 3.9. We set $d:=S \frac{\mathrm{d}}{\mathrm{dS}}$. We can assume that $f=\sum_{i \in \mathbb{N}} a_{i} S^{\frac{-i}{m}}$. Indeed, since $f=$ $f_{-}+f_{+}$with $f_{-}:=\sum_{i<0} a_{i} S^{\frac{-i}{m}}$ and $f_{+}:=\sum_{i \geq 0} a_{i} S^{\frac{-i}{m}}$, according to Remark 2.12 there exists $g \in k\left(\left(S^{\frac{1}{m}}\right)\right)$ such that $f_{+}-a_{0}=\frac{S \frac{\mathrm{d}}{\mathrm{dS}}(g)}{g}$. Therefore, we have $\left(k\left(\left(S^{\frac{1}{m}}\right)\right), \nabla\right) \simeq\left(k\left(\left(S^{\frac{1}{m}}\right)\right), S \frac{\mathrm{d}}{\mathrm{dS}}+f_{-}+a_{0}\right)$. Since the point $\pi_{F / k}(f)$ corresponds to the character $k[T] \rightarrow F, T \mapsto f$ and $F \simeq \mathscr{H}\left(x_{0, r \frac{1}{m}}\right)$, it coincides with $f\left(x_{0, r \frac{1}{m}}\right)$ (cf. Notation 1.5). Moreover, we have $f\left(x_{0, r \frac{1}{m}}\right)=x_{0,|f|}=x_{0, r^{v(f)}}$. By Lemma 3.11 $\Sigma_{f, k}\left(\mathcal{L}_{k}(F)\right)=\left\{x_{0, r v(f)}\right\}$. Let us prove now that $\Sigma_{\nabla, k}\left(\mathcal{L}_{k}(F)\right)=\left\{x_{0, r v(f)}\right\}$. Let $y \in \mathbb{A}_{k}^{1, \text { an }} \backslash\left\{x_{0, r v(f)}\right\}$. We know that $f \otimes 1-1 \otimes T(y)$ is invertible in $F \hat{\otimes}_{k} \mathscr{H}(y)$, hence invertible in $\mathcal{L}_{k}(F) \hat{\otimes}_{k} \mathscr{H}(y)$. Since $d \otimes 1=(\nabla \otimes 1-1 \otimes T(y))-(f \otimes 1-1 \otimes T(y))$, in order to prove that $\nabla \otimes 1-1 \otimes T(y)$ is invertible, it is enough to show that

$$
\|d \otimes 1\|<\left\|(f \otimes 1-1 \otimes T(y))^{-1}\right\|^{-1} .
$$

In order to do so, since $\|d\|=\|d \otimes 1\|=1$ (cf. [Azz20, Lemma 2.3] and Lemma 3.6 ), it is enough to show that $1<\left\|(f \otimes 1-1 \otimes T(y))^{-1}\right\|^{-1}$. On the one hand, since $F \hookrightarrow \mathcal{L}_{k}(F)$ is an isometric embedding, then so is $F \hat{\otimes}_{k} \mathscr{H}(y) \rightarrow \mathcal{L}_{k}(F) \hat{\otimes}_{k} \mathscr{H}(y)$ (cf. [Poi13, Lemme 3.1]). On the other hand, we have $F \hat{\otimes}_{k} \mathscr{H}(y)=\mathcal{O}\left(C_{\mathscr{H}(y)}^{+}\left(0, r^{\frac{1}{m}}, r^{\frac{1}{m}}\right)\right)$. Therefore, we have

$$
\left\|(f \otimes 1-1 \otimes T(y))^{-1}\right\|^{-1}=\|f \otimes 1-1 \otimes T(y)\|=\max \left(\left|f-a_{0}\right|,\left|T(y)-a_{0}\right|\right) .
$$

Consequently, we obtain $1<\left\|(f \otimes 1-1 \otimes T(y))^{-1}\right\|^{-1}$ in $F \hat{\otimes}_{k} \mathscr{H}(y)$, hence in $\mathcal{L}_{k}(F) \hat{\otimes}_{k} \mathscr{H}(y)$. Since the spectrum $\Sigma_{\nabla, k}\left(\mathcal{L}_{k}(F)\right)$ is not empty, we conclude that $\Sigma_{\nabla, k}\left(\mathcal{L}_{k}(F)\right)=\left\{x_{0, r v(f)}\right\}$.

Proposition 3.12. Let $(M, \nabla)$ be a differential module over $\left(k((S)), S \frac{\mathrm{d}}{\mathrm{dS}}\right)$ without regular part. The spectrum of $\nabla$ as an element of $\mathcal{L}_{k}(M)$ is:

$$
\Sigma_{\nabla, k}\left(\mathcal{L}_{k}(M)\right)=\left\{x_{0, r^{v\left(f_{1}\right)}}, \cdots, x_{0, r^{v\left(f_{N}\right)}}\right\}
$$

where the $f_{i}$ are as in the formula (3.1).

Proof. We set $\Sigma_{\nabla}:=\Sigma_{\nabla, k}\left(\mathcal{L}_{k}(M)\right)$. By Theorem 3.8, there exists $F=k\left(\left(S^{\frac{1}{m}}\right)\right)$ such

$$
\left(I_{F}{ }^{*} M, I_{F}{ }^{*} \nabla\right)=\bigoplus_{i=1}^{N} \mathscr{D}_{F} / \mathscr{D}_{F} \cdot\left(D-f_{i}\right)^{\alpha_{i}}
$$

where $f_{i} \in k \llbracket S^{-\frac{1}{m}} \rrbracket$. We set $\Sigma_{I_{F} * \nabla}:=\Sigma_{I_{F}{ }^{*} \nabla, k}\left(\mathcal{L}_{k}\left(I_{F}{ }^{*} M\right)\right)$. Since $(M, \nabla)$ is without regular part, we have $f_{i} \in k \llbracket S^{-\frac{1}{m}} \rrbracket \backslash k$. By Corollaries 2.6 and 2.7, we have:

$$
\Sigma_{I_{F}{ }^{*} \nabla}=\bigcup_{i=1}^{N} \Sigma_{S \frac{\mathrm{d}}{\mathrm{dS}}+f_{i}}\left(\mathcal{L}_{k}(F)\right) .
$$

By Proposition 3.9, we have $\Sigma_{S \frac{\mathrm{d}}{\mathrm{dS}}+f_{i}}\left(\mathcal{L}_{k}(F)\right)=\left\{x_{0, r v\left(f_{i}\right)}\right\}$. Hence,

$$
\Sigma_{I_{F}^{*} \nabla}=\left\{x_{0, r v\left(f_{1}\right)}, \cdots, x_{0, r v\left(f_{N}\right)}\right\} .
$$

By the formula (2.7), we have:

$$
\Sigma_{I_{F} * \nabla}=\bigcup_{i=0}^{m-1} \frac{i}{m}+\Sigma_{\nabla} .
$$

Since $r^{v\left(f_{i}\right)}>1$ for all $1 \leq i \leq N$, then each element of $\Sigma_{I_{F}{ }^{*} \nabla}$ is invariant by translation by $\frac{j}{m}$ where $1 \leq j \leq m$. This means that $\Sigma_{\nabla}=\Sigma_{\nabla}+\frac{j}{m}$. Therefore, we have $\Sigma_{I_{F}^{*} \nabla}=\Sigma_{\nabla}$.

Remark 3.13. Note that, it is not easy to compute the $f_{i}$ of the formula (3.1). However, the values $-v\left(f_{i}\right)$ coincide with the slopes of the differential module (cf. [Kat87] and [VS12, Remarks 3.55]). 
We now prove the main statement of the paper that summarizes all the previous results.

Proof of Theorem 3.1. According to Theorem 3.4, Proposition 3.12 and Remark 3.13 we obtain the result.

Remark 3.14. We note that, although differential modules over $\left(k((S)), S \frac{\mathrm{d}}{\mathrm{dS}}\right)$ are algebraic objects, their spectra in the sense of Berkovich depend highly on the choice of the absolute value on $k((S))$. Indeed, as en exemple we chose $\left(\mathscr{D}_{k((S))} / \mathscr{D}_{k((S))} \cdot\left(D-S^{-2}\right), D\right)$ and let $r, r^{\prime} \in(0,1)$, with $r \neq r^{\prime}$. As fields $k((S))$, $\mathscr{H}\left(x_{0, r}\right), \mathscr{H}\left(x_{0, r^{\prime}}\right)$ are the same. However, we have

$$
\Sigma_{D}\left(\mathcal{L}_{k}\left(\mathscr{H}\left(x_{0, r}\right)\right)\right)=\left\{x_{0, r^{-2}}\right\} \neq\left\{x_{0, r^{\prime-2}}\right\}=\Sigma_{D}\left(\mathcal{L}_{k}\left(\mathscr{H}\left(x_{0, r^{\prime}}\right)\right)\right)
$$

\section{Conclusion}

We may think that this result does not give more information than decomposition theorems. However, it shows clearly the existence of a link between the spectrum and the slopes of a differential module. Consequently, it leads to a connection between the spectrum and all radii of convergence.

More precisely, assume that $(k((S)),||$.$) coincides with \mathscr{H}\left(x_{0, r}\right)$ for some $r \in(0,1)$ and let $(M, \nabla)$ be a differential module over $\left(k((S)), S \frac{\mathrm{d}}{\mathrm{dS}}\right)$. Let $\gamma_{1}<\gamma_{2}<\cdots<\gamma_{\nu}$ be the positive formal slopes of $(M, \nabla)$. Then the radii of convergence of the solutions of $\left(M_{\mathrm{irr}}, \nabla_{\mathrm{irr}}\right)$ are exactly:

$$
R_{i}=r^{\gamma_{i}} ; i \in\{1, \cdots, \nu\}
$$

(cf. [Pul15, Proposition 4.3.1] and [Pul15, Section 4, (4.4)]) and satisfy $R_{1}<R_{2}<\cdots<R_{\nu}$. Consider now the spectrum $\Sigma_{\nabla}$ of $(M, \nabla)$. If $\Sigma_{\nabla} \backslash k \cup\left\{x_{0,1}\right\}=\left\{x_{0, r_{1}}, \cdots, x_{0, r_{\nu}}\right\}$, with $r_{\nu}<\cdots<r_{0}$, then we can deduce directly that the radii of convergence of the solutions of the irregular part of $(M, \nabla)$ are $R_{i}=\frac{1}{r_{i}}$ for $i \in\{1, \cdots, \nu\}$ and we have:

$$
R_{1}<R_{2}<\cdots<R_{\nu}
$$

This motivates to push the study further, and get an analogous result for a more general context. Notably, for the $p$-adic case, where the existens of $p$-adic Liouville number causes many problems namely the infinitude of de Rah cohomology. We hope that, using this spectrum, we can aviode the non-Liouville assumption. However, this notion of spectrum need to be more refined. Indeed, the multiplicity is clearly a skipped information.

\section{REFERENCES}

[Azz20] T. A. Azzouz. "Spectrum of a linear differential equation with constant coefficients". In: Mathematische Zeitschrift (Feb. 2020). ISSN: 1432-1823. DOI: 10.1007/s00209-020-02482-z. URL: http://dx.doi.org/10.1007/s00209-020-02482-z.

[Ber90] V. G. Berkovich. Spectral Theory and Analytic Geometry Over non-Archimedean Fields. AMS Mathematical Surveys and Monographs 33. AMS, 1990.

[Chr] G. Christol. "Le théorème de turritin p-adique (version du 11/06/2011)".

[CM00] G. Christol and Z. Mebkhout. "Sur le Theoreme de l'Indice des Equations Differentielles pAdiques III". In: Annals of Mathematics 151.2 (2000), pp. 385-457. ISSN: 0003486X. URL: http://www.jstor.org/stable/121041.

[CM01] G. Christol and Z. Mebkhout. "Sur le théorème de l'indice des équations différentielles padiques IV". In: Inventiones mathematicae 143.3 (Mar. 2001), pp. 629-672. ISSN: 1432-1297. DOI: $10.1007 /$ s002220000116. URL: http://dx.doi.org/10.1007/s002220000116.

[DMR07] P. Deligne, B. Malgrange, and J. Ramis. Singularités irrégulières. Correspondance et documents. French. Vol. 5. Paris: Société Mathématique de France, 2007, pp. x + 188. ISBN: 978-2-85629-241-9/hbk. 
[DR77] B. Dwork and P. Robba. "On Ordinary Linear p-Adic Differential Equations". In: Transactions of the American Mathematical Society 231.1 (1977), pp. 1-46. ISSN: 00029947. URL: http://www. jstor.org/stable/1997866.

[Kat70] N. M. Katz. "Nilpotent connections and the monodromy theorem: Applications of a result of Turrittin." English. In: Publ. Math., Inst. Hautes Étud. Sci. 39 (1970), pp. 175-232. ISSN: 0073-8301; 1618-1913/e. DOI: $10.1007 /$ BF02684688.

[Kat87] N. M. Katz. "On the calculation of some differential galois groups". In: Inventiones mathematicae 87.1 (Feb. 1987), pp. 13-61. ISSN: 1432-1297. DOI: 10.1007/BF01389152. URL: https://doi.org/10.1007/

[Ked10] K. S. Kedlaya. p-adic differential equations. English. Cambridge: Cambridge University Press, 2010, pp. xvii + 380. ISBN: 978-0-521-76879-5/hbk.

[Ked13] K. S. Kedlaya. "Local and global structure of connections on nonarchimedean curves". In: ArXiv e-prints (Jan. 2013). arXiv: 1301.6309 [math.NT].

[Mal74] B. Malgrange. "Sur les points singuliers des équations différentielles". In: Enseignement Math. (2) 20 (1974), pp. 147-176. ISSN: 0013-8584.

[Poi13] J. Poineau. "Les espaces de Berkovich sont angéliques". In: Bull. soc. Math. France (2013).

[Pul15] A. Pulita. "The convergence Newton polygon of a $p$-adic differential equation. I: Affinoid domains of the Berkovich affine line." English. In: Acta Math. 214.2 (2015), pp. 307-355. ISSN: 0001-5962; 1871-2509/e. DOI: 10.1007/s11511-015-0126-9.

[Rob75a] P. Robba. Lemme de Hensel pour les opérateurs différentiels. French. Groupe d'Etude d'Anal. ultrametr., 2e annee 1974/75, Expose 16, 11 p. (1975). 1975.

[Rob75b] P. Robba. "On the Index of p-adic Differential Operators I". In: Annals of Mathematics 101.2 (1975), pp. 280-316. ISSN: 0003486X. URL: http://www. jstor.org/stable/1970992.

[Rob75c] Philippe Robba. "Lemme de Hensel pour les opérateurs différentiels". fr. In: Groupe de travail d'analyse ultramétrique 2 (1974-1975). talk:16. URL: http://www. numdam. org/item/GAU_1974-1975__2__A14

[Tur55] H. L. Turrittin. "Convergent solutions of ordinary linear homogeneous differential equations in the neighborhood of an irregular singular point". In: Acta Math. 93 (1955), pp. 27-66. DOI: 10.1007/BF02392519. URL: https://doi.org/10.1007/BF02392519.

[VS12] M. Van der Put and M. F. Singer. Galois Theory of Linear Differential Equations. Grundlehren der mathematischen Wissenschaften. Springer Berlin Heidelberg, 2012. ISBN: 9783642557507.

Tinhinane Amina, AZZOUZ

Univ. Grenoble Alpes, CNRS, Institut Fourier, F-38000 Grenoble, France

Current address: Université Alger 1, 02 Rue Didouche Mourad, Algiers, Algeria

E-mail address: t.azzouz@univ-alger.dz 